\title{
Public transport traffic management systems simulation in Craiova city
}

\author{
Ilie Dumitru \\ Assoc. Prof., University of Craiova, Faculty of Mechanics, Romania \\ Lucian Matei \\ Assist. Prof., University of Craiova, Faculty of Mechanics, Romania \\ Laurentiu Racila \\ Assoc. Prof., University of Craiova, Faculty of Mechanics, Romania \\ Dumitru Nicolae
}

\begin{abstract}
Urban transport is a comprehensive and dynamic mechanism. Therefore, all the problems for improving and reorganization of the system can be examined only in the light of a systemic approach. Currently, public passenger transport is one of the most important branches of the urban development in cities and metropolis. Public passenger transport activity and all the steps taken to improve that activity are considered to be of great social importance. In the current stage of city development, one of the main tasks is to create a public passenger transportation system that is safe, affordable, economical, reliable and environmentally friendly. The important role of passenger transport in the city's economy and achieving important social services to the population, dictates the need to introduce measures in the system that are harmonious, balanced and effective. This can only be done, in the context of current development, only after the system as a whole is tested extensively through special traffic and management software.
\end{abstract}

\section{INTRODUCTION}

The population mobility that is traveling in public urban transport can be considered as the number of trips by public transport in relation to city residents during the year [1], according to the relationship:

$$
M_{t r}=\frac{Q}{N_{p o p}}
$$

where:

$\mathrm{Q}$ - is the number of passengers transported during one year, in thousand passengers;

$\mathrm{N}_{\text {pop }}$ - urban population, thousand persons;

The formula offers the possibility (taking as input No. of trips / passenger / year) to determine the opportunity to modernize the public transport with minimum inconvenience [2]. It is extremely difficult to take into account a whole city: transport, freight vehicle, personal vehicles, traffic behaviour, traffic lights, road surface quality, the geometry of streets and intersections, angles of vision, pedestrians, etc [2,3]. We can put the problem in the following way: if you change a bus stop after an intersection according to the theory of 
complex systems my nonlinear dynamic system will present a instability phenomenon called the sensitivity to initial conditions, so that relatively long-term repercussions (although complying with deterministic laws) is unpredictable.

An important aid in order to achieve an optimization of public transport at an entire city as well at an intersection (which will take into account multiple factors which belong at the city level) are modelling and simulation software's for road transport [4].

\section{CONTEXT}

\subsection{Simulation and modelling of the public transport in Craiova}

Since the trend in Europe and worldwide is to discourage individual road transport in favour of public transport, the necessary measures to increase the attractiveness of the latter needs to be much more obvious, especially effective in this regard (increase comfort, traffic safety, insurance transmission capacity while ensuring an appropriate degree of regularity etc.). In light of these problems the objectives of this paper are the following [5]:

- Create a virtual model of the main arteries of Craiova

- Modelling the current situation of the public transport in the Craiova city's main arteries

- Green light for public transport in areas where street network allows

\subsection{Virtual model}

To realize a virtual transport network for the city of Craiova we used a simulation and modelling program called Aimsun. Based on current research on public transport in Craiova was concluded that modelling the entire city is not required, since most activity transport routes of the city is developed (by intersecting and overlapping) on the main roads [6]. Due to this thing we modelled in Aimsun only the city's main transport networks (which confound with the main arteries) so virtual system consisting of $85 \mathrm{~km}$ long section, $139 \mathrm{~km}$ long lane, 762 sections, 269 junctions, 55 centroids, 103 stations for public transport (Figure 1).
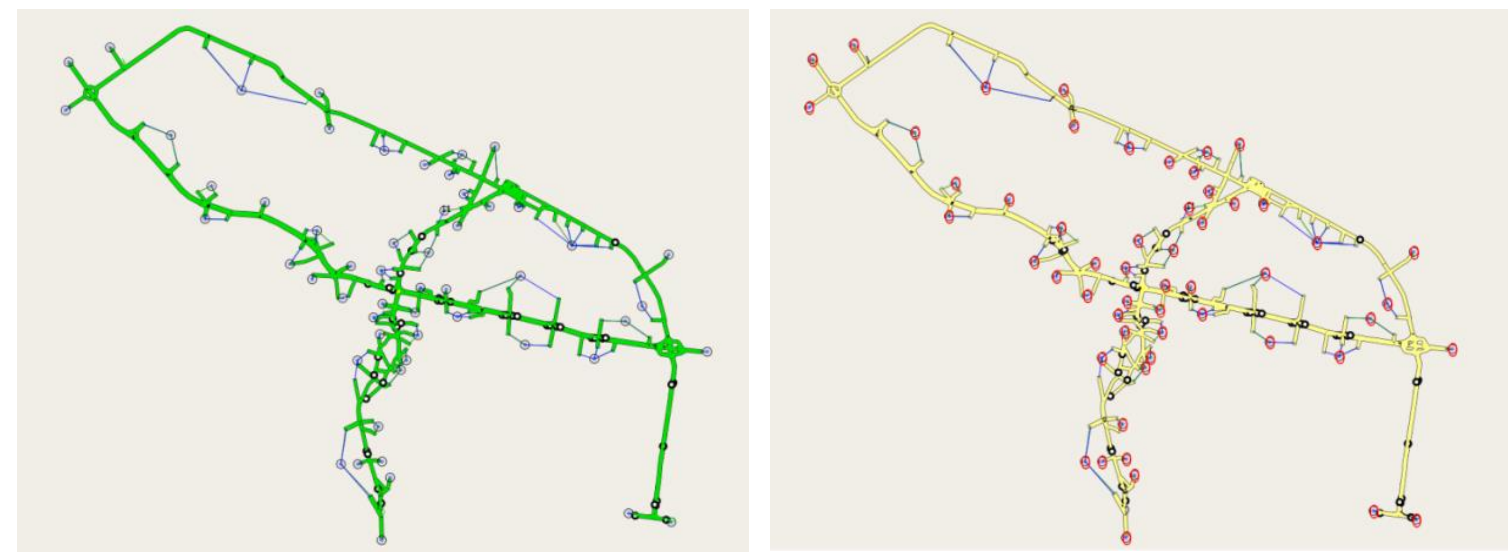

Fig. 1 - Craiova city main arteries and model centroids

One of the problems in a system so great is that a real origin / destination array of the entire 
city is very difficult to create. In most cases the traffic measurements is performed at key points then, based on these measurements, using mathematical models we can achieve a good origin / destination matrix [6,7]. Aimsun can generate this matrix alone, based on a mathematical model of Wardrop. Very important at this stage is detector array configuration which will generate Aimsun origin / destination matrix. Because the model is in an early stage, it is necessary to realize allocation of routes that vehicles in the network use based on various parameters:

- Cost

- Waiting time

- The traffic volume

- Vehicle Classes

Once the routes allocation is done for each vehicle in the Aimsun virtual network, we go to origin / destination matrix generation based on information contained in the detection and information generated during route allocation. Modelled transport system for the city of Craiova contains all bus and tram routes in Craiova, because currently there is no path that does not have a few main station on the main roads [7].

For the configuration of the public transport is not required an origin / destination matrix as we know the exact configuration and waiting time at the station, route, public stops of the public transport vehicles (Figure 2).

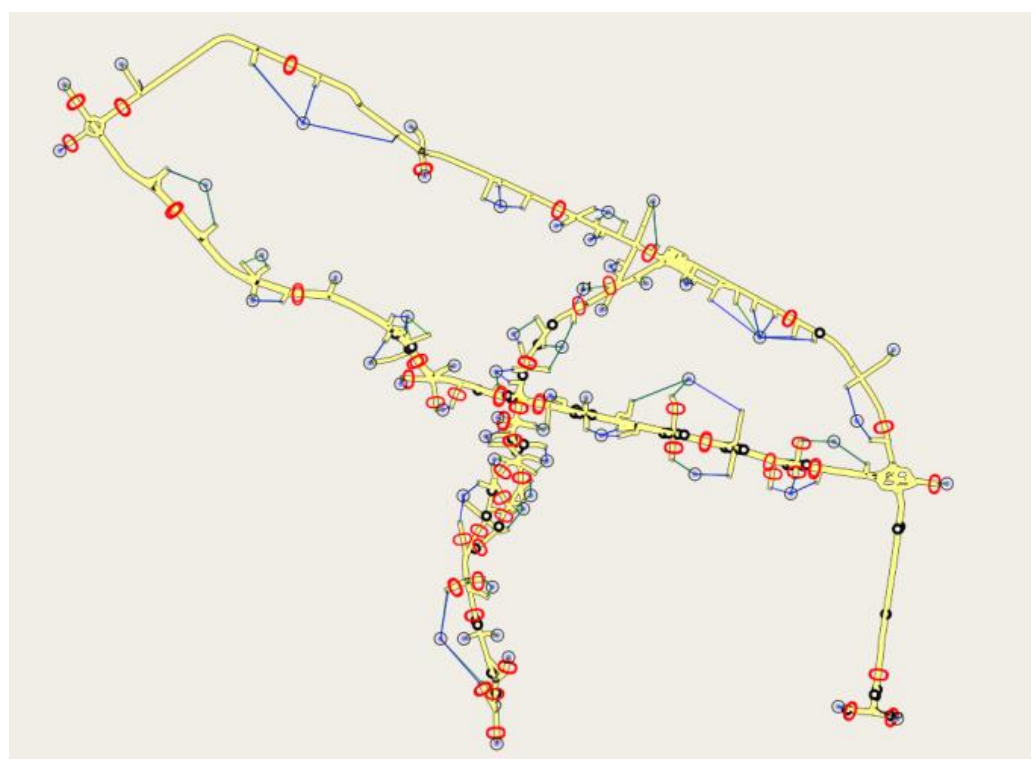

Fig. 2 - Public station stops in the network model

Configuration of transport system involves simulating real conditions that make up the fleet of vehicles for public transportation. These conditions do not affect the actual fleet itself, but the way the created Aimsun model works (Figure 3).

Because the transport system modelling for Craiova assumed introduction of parameters that make Aimsun transport network, the parameters are fixed, their configuration is not necessary. At the time when the calibration was done for the city's main arteries, a public transport system calibration was performed automatically because it is dependent on the 
structure of streets and the general parameters of traffic $[7,8]$.

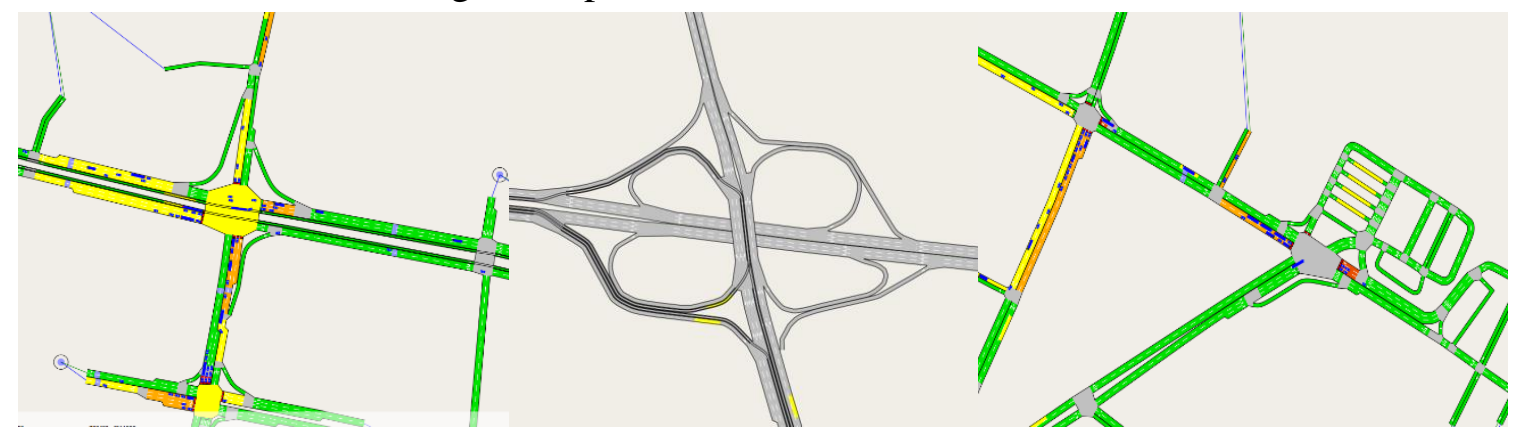

Fig. 3 - Street and intersection geometry in AIMSUN model

\subsection{Priority simulation for public transport}

Green light priority is a method of optimizing the transport and aims to reduce waiting time in traffic for transport vehicle so it will increase security, will decrease the time spent in the in traffic from the point of view of the passenger, the passenger discomfort for the duration of the journey etc.

To create the green light priority transport were added to every traffic light, detectors. This acts as inductive loop detectors and detects vehicles transport. For this detection to operate in optimum safety, transport vehicles that run on the main arteries of the city Craiova must be equipped with special tools that the inductive loop can recognize them so they can send signals to the automatic traffic controller that the traffic light currently plan will be interrupted by a new plan. The new plan involves in clearing the current lane. When the detector has detected a public transport vehicle it will change the current signal traffic light in green that the vehicles before the public transport vehicles can clear the lane so the priority for the public transport can be achieved [8].

These detectors must be entered into the system in pairs of two so that one function as inductive loop detecting the public transport vehicle and give the command to change the automatic traffic signal plan and after the intersection to detect the public transport vehicle that exits the intersection and send the signal to the automatic traffic controller to change the signal to the one used before detection.

The simulation results can be seen in the following figures:

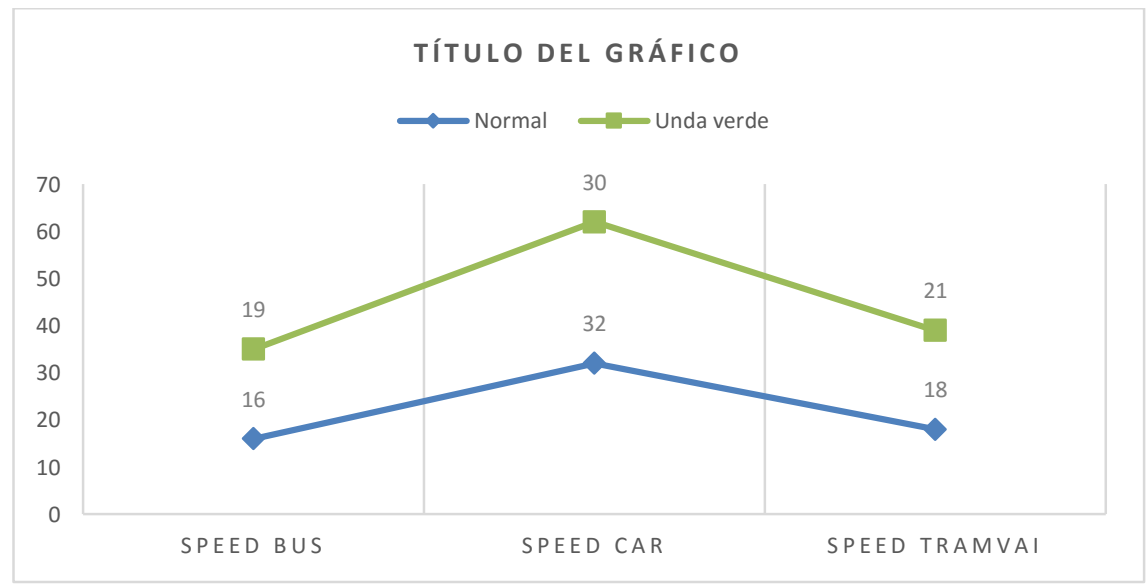


Fig. 4 - Comparison between delay times

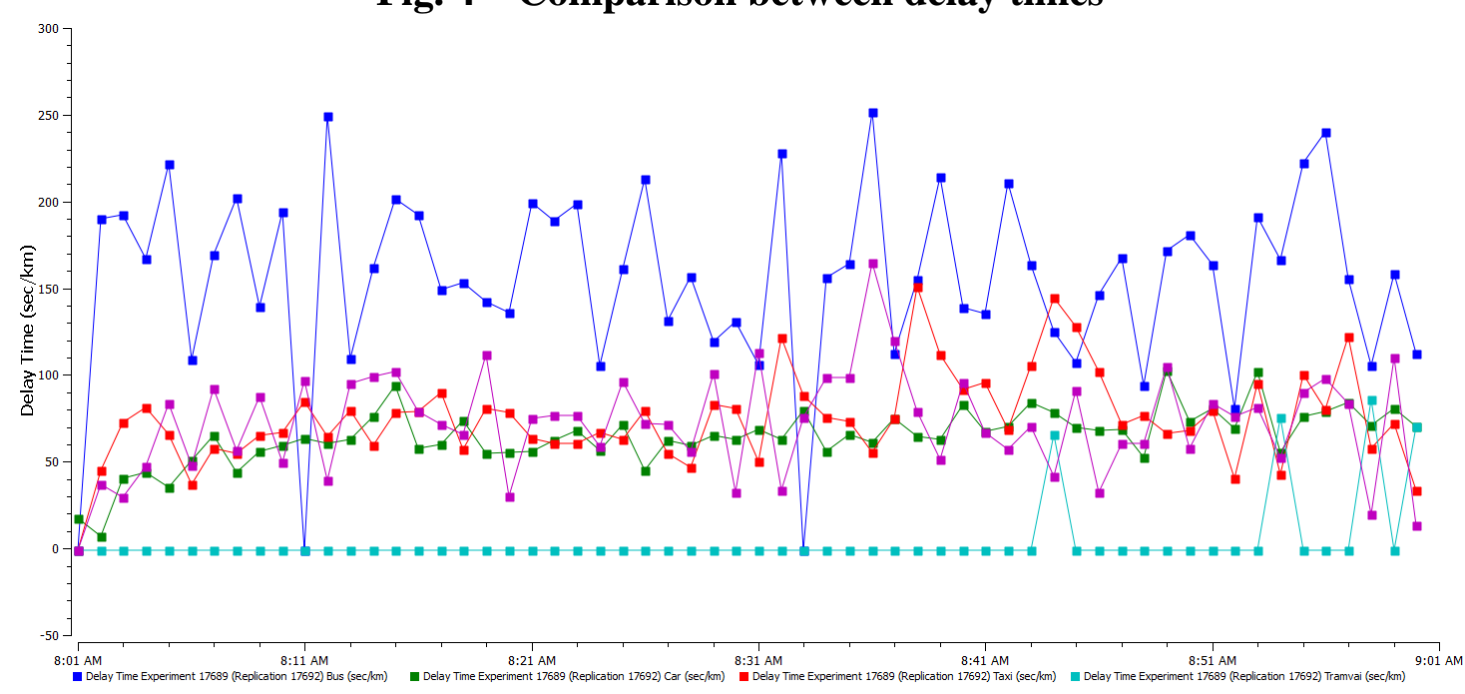

Fig. 5 - Delay time - green wave simulation

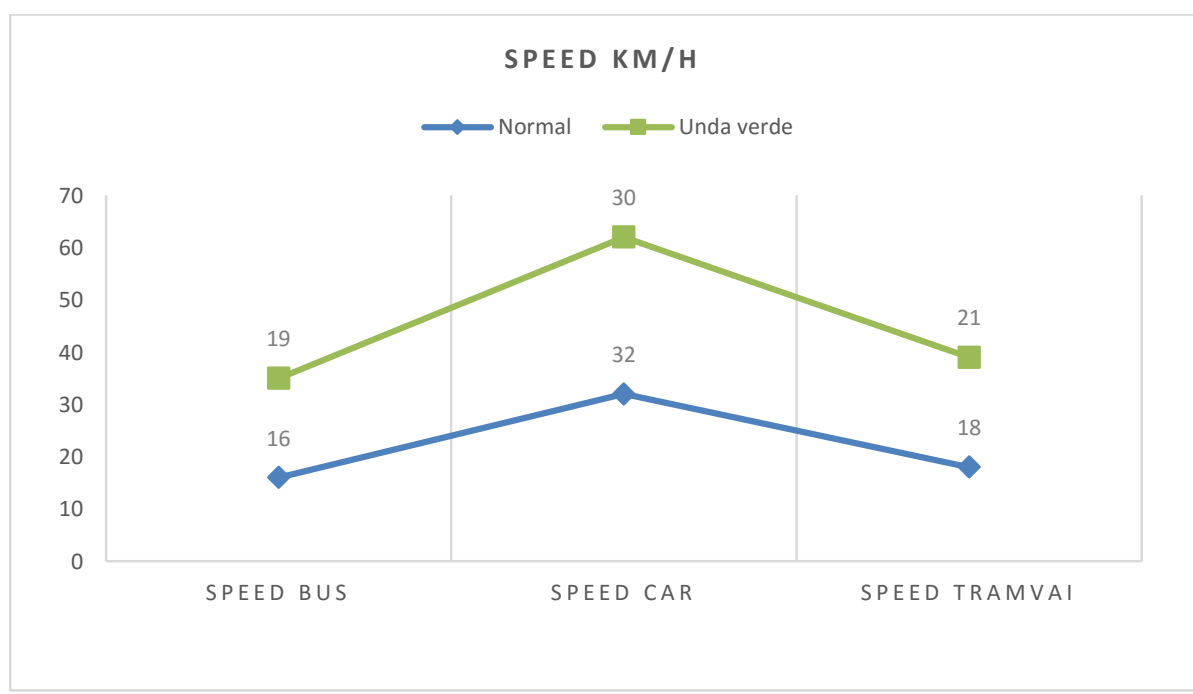

Fig. 6 - Speed comparison

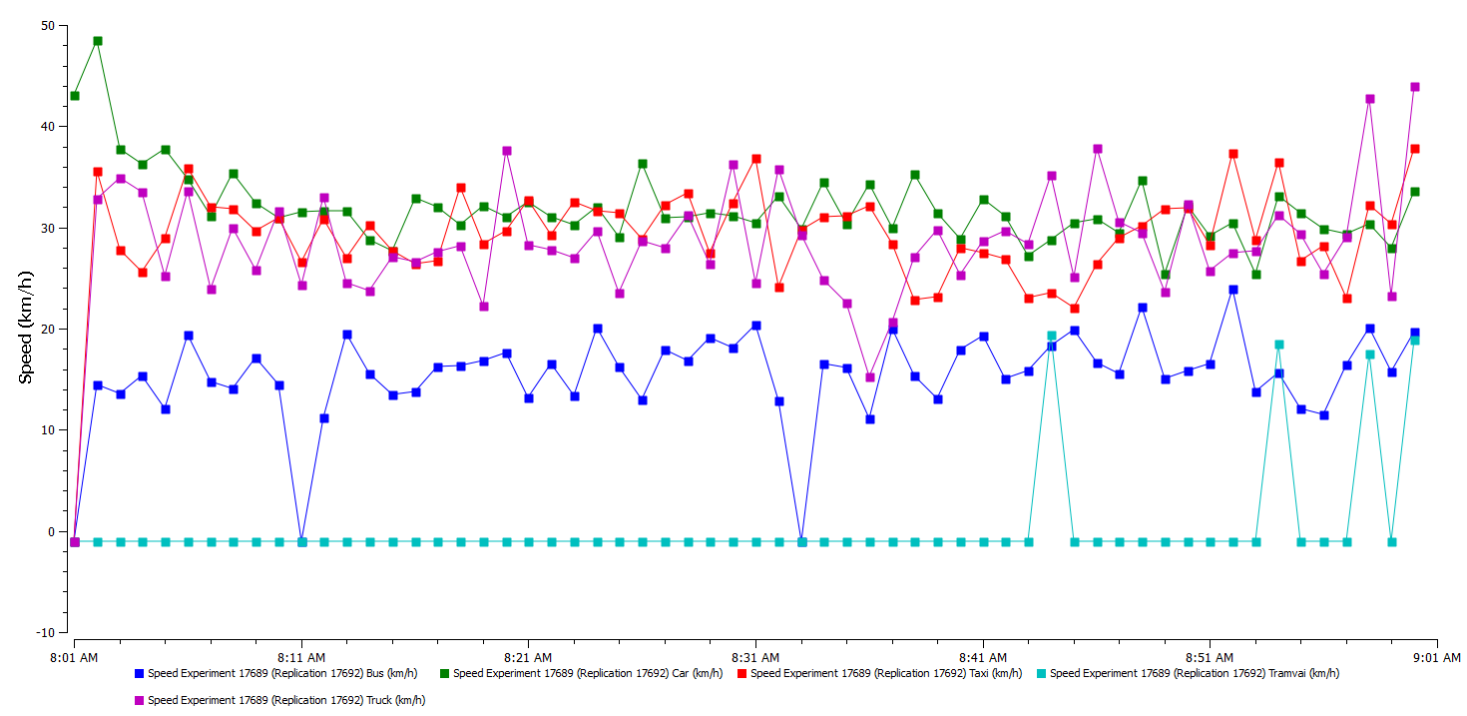

Fig. 7 - Speed - green wave simulation 


\section{CONCLUSION}

Craiova has a well-developed public transport network which covers the entire city and that provides a comfortable ride from home to areas of interest. There are opportunities for providing solutions in the virtual environment on the decongestion of traffic and prioritization of public transport that allow eco efficiency for road traffic in any urban agglomeration.

The Figure 4 shows that traffic delay time decreases to buses and trams for $30 \mathrm{sec} / \mathrm{km}$ when the green light prioritization is introduced into the network transport on main roads in the city of Craiova. A disadvantage of these measures can be the increased delay time in light vehicle traffic by $23 \mathrm{sec} / \mathrm{km}$. Figure 5 shows a detailed map of the delay time in the studied model for all the vehicle classes used.

Another improvement is the cruise speed of public transport in the city's main arteries. One can see a rather striking increase for in speed for the peak hour in public transport such as trams and buses plotted in Figure 6 and a more detailed one in Figure 7.

Based on additional studies and traffic measurements we can easily determine the critical points of the city in which priority band and green light prioritization can be a huge success so as to increase the quality, safety and attractiveness of public transport. So depending on the particular situation we can prepare and develop a new and private research methodology. This approach (modelling and simulation prioritization tram) meets with tram modernization of public transport, given that 2014 is over, replacement and modernization of the runway.

\section{REFERENCES}

[1] C.M. Andrews, S.M. Elahi, J.E. Clark, Traffic-Control System In Transportation Research Record, pp. 150-155, 1998.

[2] Treiber, Martin and Dirk Helbing, "Microsimulation of Freeway Traffic Including Control Measures", (2002).

[3] Boyer, Kenneth D., „Principles of Transportation Economics”, Addison Wesley Longman publishing house, 1999.

[4] Dowling, R., ,Definition, interpretation, and calculation of traffic analysis tools measures of effectiveness", Federal Highway Administration, Washington DC, 2007

[5] J. A. Stewart, M.V. Aerde, An Assessment of Adaptive Co-ordination of Traffic Signal offsets within integration, Traffic Engineering and Control, Volume 39, 1998.

[6] Matei, L., Dumitru I., Racilă, L., Vînatoru, M., Adaptive traffic signal control on a national road intersection Applied Mechanics and Materials, ISSN: 1662-7482, Vol. 822, pp $455-460$

[7] OȚĂT Oana Victoria; DUMITRU Ilie; OŢĂT Victor; MATEI Lucian, The role of infomobility systems in optimizing the public transport. Case study Craiova, Congress AMMA 2013 - Automotive Motor Mobility Ambient, 17-19 october, Cluj - Napoca

[8] Highway Capacity Manual 2010, Transportation Research Board, American Association of State Highway and Transportation Officials (AASHTO) and Federal Highway Administration, 2010. 